\title{
Distraction osteogenesis and arthrodesis as a new surgical option for chondrosarcoma in the distal tibia
}

\author{
Zhengxiao Ouyang, Xuezheng Xu, Linqing Li, Yi Luo, Jianfan Liu, Xin Wang, Xinyu Yao, Gang Huang \\ and Xianan Li
}

\begin{abstract}
Recent advances in the management of bone tumors have led to a significant increase in the survival rates of patients with malignant bone tumors. Thus, limb salvage surgery has gained importance for preserving limb function in the management of bone tumors. However, surgery presents unique difficulties in terms of the biomechanics and obtaining a soft-tissue cover, such as when the ankle is involved in the primary malignant bone tumor. We report a case of chondrosarcoma of the distal tibia treated with wide en bloc resection arthrodesis and reconstruction of the defect using distraction osteogenesis, which offers an effective alternative protocol for limb salvage. The patient has remained disease free for 3 years since the initial surgery and can maintain normal limb athletic function.
\end{abstract}

Keywords: Limb salvage, Chondrosarcoma, Distraction osteogenesis, Bone lengthening, Arthrodesis

\section{Background}

Recent advances in the management of bone tumors, including preoperative radiological evaluation, chemotherapy, materials and implant technology, and surgical technique, have led to a significant increase in the survival rates of patients with malignant bone tumors [1-3]. Therefore, limb salvage surgery has gained importance for providing adequate tumor resection while preserving a functional limb $[2,4,5]$. Various methods, including biological (e.g., vascularized autograft, allograft, recycled bone treated by radiation, autoclaving, pasteurization, liquid nitrogen, and distraction osteogenesis) and nonbiological (prosthesis) methods, have been established for the reconstruction of bone defects after malignant or benign bone tumor excision; however, a gold standard method for reconstruction does not exist $[2,3,6]$, and there are few reports of reconstruction of large bone defects in the distal tibia following wide resection of malignant bone tumors, adding to the challenge of limb salvage surgery.

\footnotetext{
* Correspondence: lixianan2001@163.com

Department of Orthopaedics, Hunan Cancer Hospital and The Affiliated

Cancer Hospital of Xiangya School of Medicine, Central South University, 283 Tongzipo Road, Changsha, Hunan 410012, China
}

This report describes a patient with chondrosarcoma of the distal tibia who underwent limb salvage surgical resection followed by limb lengthening and arthrodesis. There are no reports of the use of this combined approach to produce limb salvage and a functional lower extremity. The patient received a detailed explanation concerning the surgical procedure and the intent to submit data from the case for publication, and he provided consent.

\section{Case presentation}

A 23-year-old man presented with left leg pain. Radiographs revealed an aggressive lesion of the distal tibia (Fig. 1). Biopsy (Fig. 2) and appropriate staging studies confirmed stage IIB chondrosarcoma [7]. He was treated with wide en bloc resection, including the distal third of the tibia and the cartilage of the talus in the tibiotalar joint. He then underwent a distal tibia lengthening with a unilateral external fixator (Fig. 3). Gradual distraction started 5-7 days after surgery and was applied at a rate of $1 \mathrm{~mm}$ per 36 hours, and $8 \mathrm{~cm}$ of length was gained and corticalized over a 6-month period. Fusion of the tibiotalar joint was then allowed to proceed for 3 months before removal of external fixation (Fig. 4). The current 


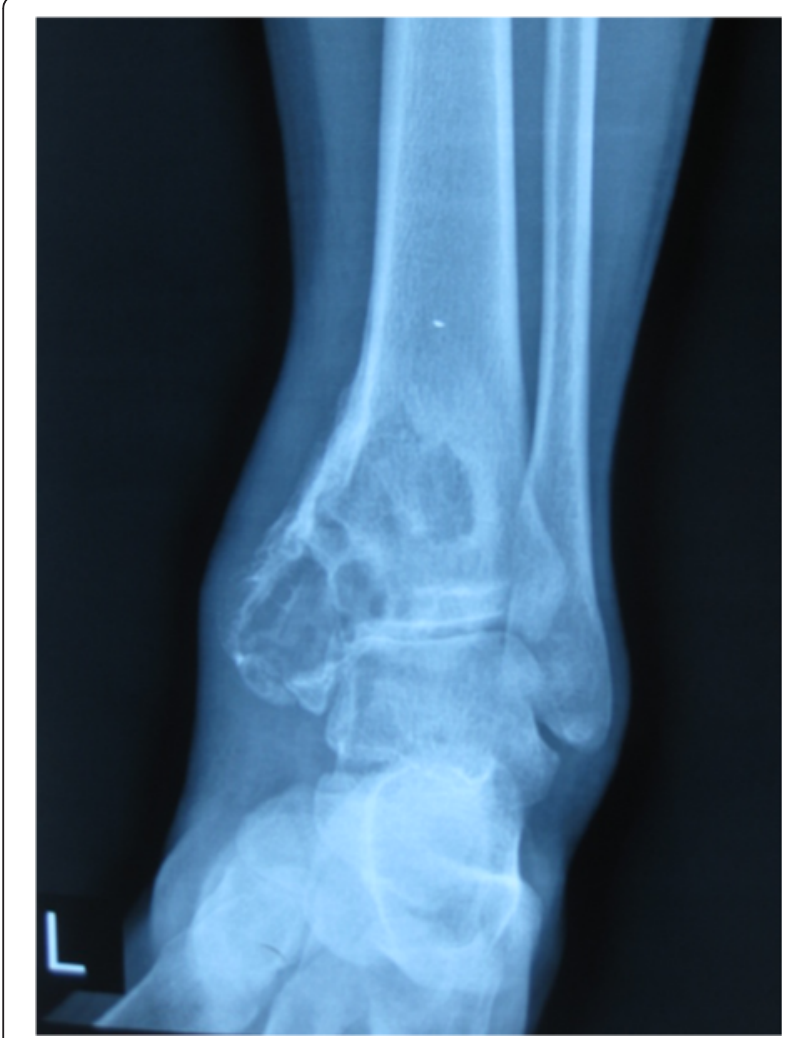

Fig. 1 Radiographs revealing an aggressive lesion of the distal tibia

limb-length discrepancy is minimal (Fig. 5). He has remained disease free for 3 years since the initial surgery and can maintain normal limb athletic function.

The external fixation index calculated by dividing the entire duration of external fixation by the length of bone regeneration was 36 days $/ \mathrm{cm}$; the distraction index calculated by dividing the duration of distraction by the length of bone regeneration was 11.08 days $/ \mathrm{cm}$, and the

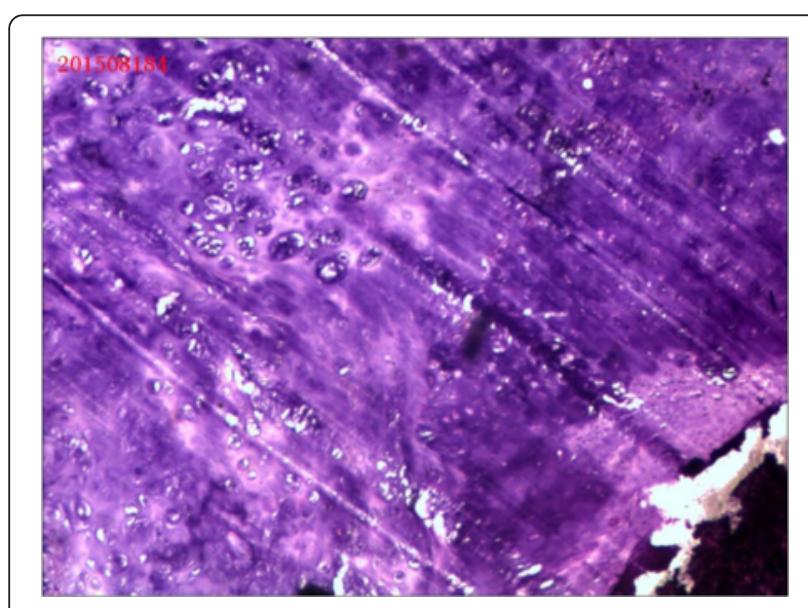

Fig. 2 Photomicrographs of the lesion maturation index calculated by dividing the duration of external fixation by the length of bone regeneration was 25.8 days $/ \mathrm{cm}$, as measured from the completion of distraction to the removal of external fixation. The patient was evaluated via physical examinations and radiographs throughout the follow-up period. The function of the affected limb was assessed according to the revised 30-point functional classification system established by the International Society of Limb Salvage and the Musculoskeletal Tumor Society (MSTS) [7]. The MSTS score was 94 at the final follow-up, and the Foot and Ankle Disability Index (FADI) for patients with a tumor around the ankle joint was 90.

\section{Discussion}

Limb salvage surgery is currently a very commonly performed procedure [8]. However, restoration and longterm maintenance of good limb function after resection of a bone tumor remain a challenge. The ideal reconstruction should have biological affinity and infection resistance. More importantly, for weight-bearing lower extremities, sufficient biomechanical strength and durability are indispensable [9-11]. At present, there is no gold standard method for reconstruction [2, 3, 6], and there are few reports of reconstruction of large bone defects in the distal tibia following wide resection of malignant bone tumors, adding to the challenge of limb salvage surgery.

After considering the difficulties in determining the best treatment strategy for the current patient, another hospital suggested amputation after open biopsy for the following reasons: (1) amputation has been the standard surgical treatment, with satisfactory functional results using an appropriate prosthesis [12], and (2) difficulties exist both in terms of the biomechanics and obtaining a soft-tissue cover [13]. For such a young patient, return to function and preservation of cosmetic appearance are of great importance, especially considering his need to make social and financial plans for the prolonged rehabilitation period. Furthermore, patients treated with limb salvage surgery for distal lower leg sarcoma had excellent final functional results without impairing the oncologic results [14]. This is also favorable because (1) there are fewer musculotendinous attachments in the distal tibia than elsewhere, and (2) these attachments often contribute to the tumor breaking through the compartment.

Thus, we decided to perform limb salvage surgery. In this case, the tumor extended into the meta-epiphysis; thus, intra-articular resection including the articular surface was required. Under these circumstances, reconstructive options present unique difficulties. Endoprosthetic replacement has been reported to possess many advantages, including early stability, mobilization, 


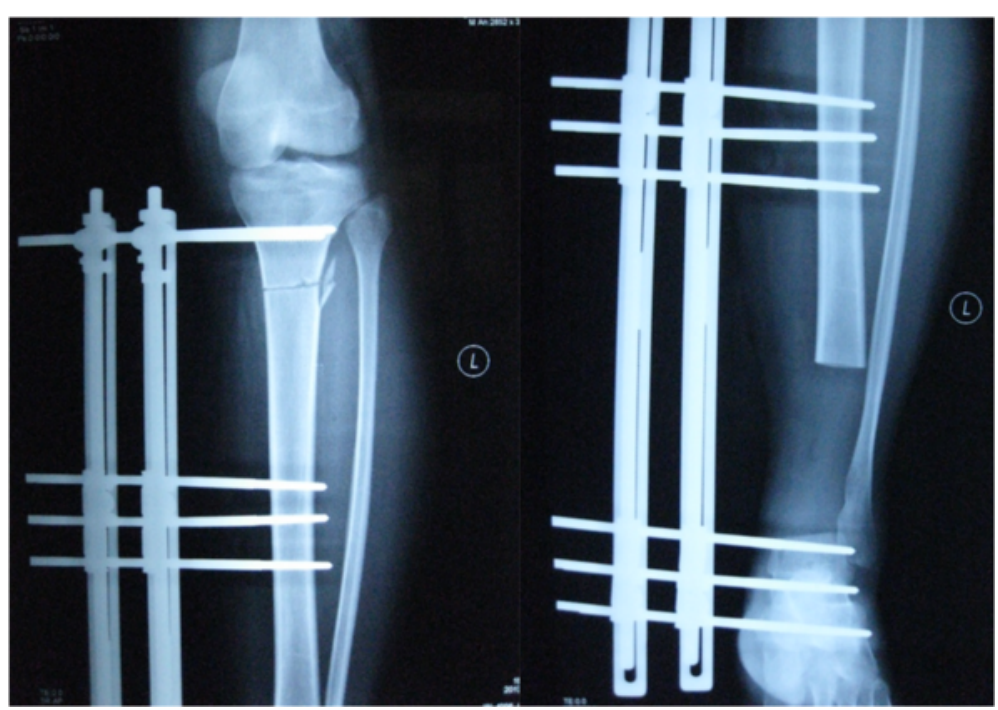

Fig. 3 Distal tibia lengthening with a unilateral external fixator after excision of the tumor

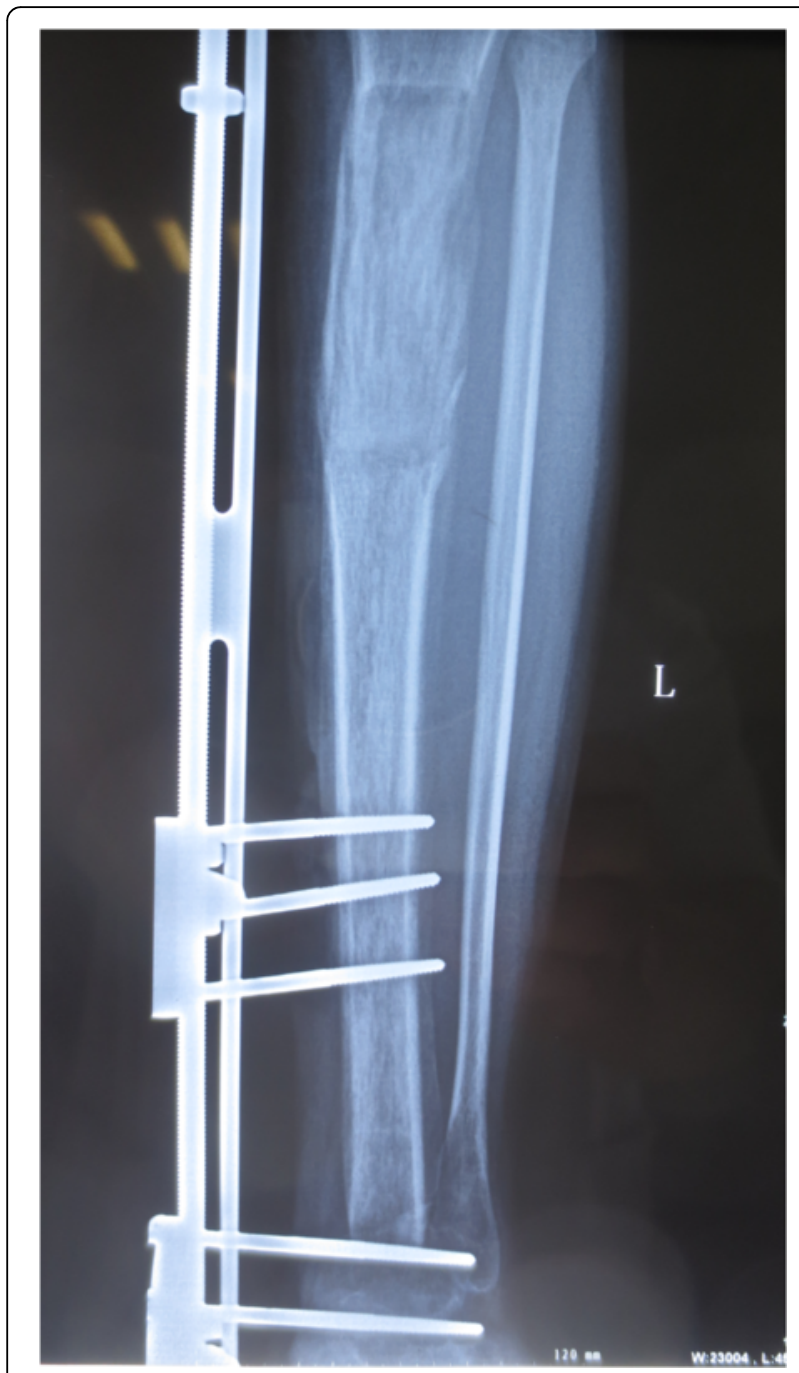

Fig. 4 Ankle joint arthrodesis after bone lengthening weight-bearing, and rapid restoration of function with a good functional outcome; however, problems such as infection, mechanical failure, and aseptic loosening exist and may limit the long-term survival of the prosthesis, increasing the risk of revision over time [12, 15]. In addition, the lack of soft tissue after wide resection might add to the risk of infection and ultimately amputation. For allografts, there are high rates of complications such as nonunion [16], infection, fracture, degeneration of the articular surface, graft resorption, joint instability, and pathological fractures [16-19]; accordingly, allografts are considered a temporary solution in the management of malignant bone tumors [20]. Moreover, postoperative chemotherapy delays incorporation and union because of negative effects on healing and revascularization [21]. Generally, complications gradually increase over time in limbs reconstructed with tumor prostheses or allografts, and limb function also worsens.

Distraction osteogenesis has been widely used as a biological approach for repairing segmental bone defects $[22,23]$ and can regenerate living bone of sufficient strength; thus, it can preserve limb function over a lifetime [24, 25]. Excellent results have been reported [1, 26-29] for distraction osteogenesis in reconstruction after massive bone loss due to tumor resection, and it has been concluded to be beneficial in patients with an expectation of long-term survival. The indications for bone distraction are (1) stage IIB malignant bone tumors when chemotherapy is judged to be effective and an epiphysis could be preserved or (2) low-grade or aggressive benign bone tumors [1]. However, none of these previous studies reported results when the ankle was involved. In the ankle, good functional and oncological results have been reported for arthrodeses with autogenous fibular strut 


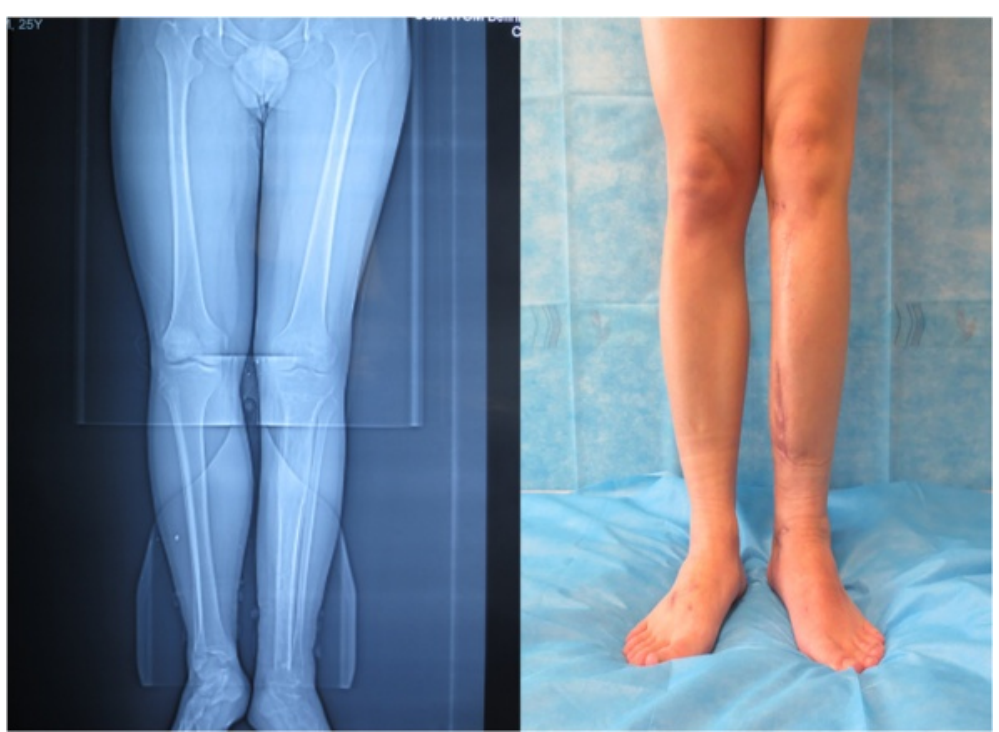

Fig. 5 Nine months after surgery, the affected limb was similar to the unaffected side in length, form, and function

grafts stabilized using an Ilizarov external fixator [30] and arthrodeses with autogenous bone grafts [13,31,32]. However, the period for graft union is long, especially in patients with non-vascularized grafts (18 months) [30].

In our opinion, there are at least five reasons for choosing distraction osteogenesis and joint fusion for patients with malignant tumors in the distal tibia. First, retaining most of the original tibia and sufficient epiphysis thickness permits full weight bearing. Second, patients with malignant bone tumors in such locations have a favorable prognosis [14]. Third, the same amount of bone ossification as seen in distraction osteogenesis during reconstruction has not been observed in vascularized fibulae [26]. Fourth, the need for bone banking, donor site morbidity, and the risk of disease transmission associated with allografts are eliminated [33]. Moreover, our combination of surgical options prevented the patient from walking for just 9 months.

Disadvantages in bone distraction include delayed union at the docking site and pin- or wire-tract infection. However, such complications were not observed in this case. Once function has been restored, it can be maintained throughout life, without anxiety concerning loosening or revision. The use of distraction osteogenesis in the treatment of infection and tumors is well established but has not been reported in treating severe bone loss after tumor resection at the ankle region. Here, the indication of bone distraction was extended to include such a case in which the epiphysis could not be preserved.

Several points should be noted in the application of such surgical procedures. (1) There is an abrupt increase in the complication rate during bone lengthening of the tibia in patients with bone defects $\geq 15 \mathrm{~cm}$ after tumor excision; this is due to the greater length of time required [34, 35]. Some researchers have recommended that such cases should be excluded [36]. In contrast, another group reported different results [26]. (2) Charnley was the first to describe compression ankle arthrodesis using a uniplanar external fixator. Since then, many types of fixators have evolved to improve fixation stability [37], including the excellent representative Ilizarov apparatus. However, the choice of technology depends on many factors such as the surgeon's familiarity and the economic status of the institution. (3) Our patient was not simultaneously undergoing chemotherapy during the distraction period, so we are unable to comment on the use of distraction osteogenesis with these treatments. However, it is reported that chemotherapy has no hazardous effect on bone regeneration with distraction osteogenesis $[26,38]$ if regional blood flow is maintained within the normal range.

\section{Conclusions}

In general, the choice of reconstructive procedure should be considered with several factors, such as the site and involvement of the tumor, pathology and biological behavior of the tumor, life expectancy, and predicted function of the limb. We believe that wide en bloc resection arthrodesis, with reconstruction of the defect using distraction osteogenesis, offers an effective alternative protocol for limb salvage in cases of chondrosarcoma of the distal tibia. Though reconstruction using bone distraction requires both time and effort, it can provide excellent long-term outcomes, resulting in a stable reconstruction that functionally restores the natural limb and is cosmetically appealing. 


\section{Consent}

Written informed consent was obtained from the patient for publication of this case report and any accompanying images. A copy of the written consent is available for review by the editor-in-chief of this journal.

\section{Competing interests}

The authors declare that they have no competing interests.

\section{Authors' contributions}

$\mathrm{ZO}$ contributed to acquisition and analysis of data and drafting of the work and surgery. XX contributed to acquisition of data and surgery. LL contributed to drafting of the work and surgery plan. YL contributed to revision of the work. JL contributed to analysis of data. XW contributed to study conception. $X Y$ contributed to acquisition of data. GH revised the work. $\mathrm{XL}$ designed the surgery and gave final approval and took overall responsibility for the published work. All authors read and approved the final manuscript.

\section{Acknowledgements}

This study was conducted without funding.

Received: 16 February 2015 Accepted: 13 May 2015

Published online: 22 May 2015

\section{References}

1. Watanabe K, Tsuchiya H, Yamamoto N, Shirai T, Nishida H, Hayashi K, et al. Over 10-year follow-up of functional outcome in patients with bone tumors reconstructed using distraction osteogenesis. J Orthop Sci. 2013;18:101-9.

2. Grimer RJ. Surgical options for children with osteosarcoma. Lancet Oncol. 2005;6:85-92.

3. Kunz P, Bernd L. Methods of biological reconstruction for bone sarcoma: indications and limits. Recent Results Cancer Res. 2009;179:113-40.

4. Messerschmitt PJ, Garcia RM, Abdul-Karim FW, Greenfield EM, Getty PJ. Osteosarcoma. J Am Acad Orthop Surg. 2009;17:515-27.

5. Heare T, Hensley MA, Dell'Orfano S. Bone tumors: osteosarcoma and Ewing's sarcoma. Curr Opin Pediatr. 2009;21:365-72.

6. Erler K, Yildiz C, Baykal B, Atesalp AS, Ozdemir MT, Basbozkurt M. Reconstruction of defects following bone tumor resections by distraction osteogenesis. Arch Orthop Trauma Surg. 2005;125:177-83.

7. Enneking WF, Dunham W, Gebhardt MC, Malawar M, Pritchard DJ. A system for the functional evaluation of reconstructive procedures after surgical treatment of tumors of the musculoskeletal system. Clin Orthop Relat Res. 1993;241-246.

8. Bacci G, Ferrari S, Lari S, Mercuri M, Donati D, Longhi A, et al. Osteosarcoma of the limb. Amputation or limb salvage in patients treated by neoadjuvant chemotherapy. J Bone Joint Surg Br. 2002;84:88-92.

9. Abed YY, Beltrami G, Campanacci DA, Innocenti M, Scoccianti G, Capanna R. Biological reconstruction after resection of bone tumours around the knee: long-term follow-up. J Bone Joint Surg Br. 2009;91:1366-72.

10. Tsuchiya H, Tomita K, Minematsu K, Mori Y, Asada N, Kitano S. Limb salvage using distraction osteogenesis. A classification of the technique. J Bone Joint Surg Br. 1997;79:403-11.

11. Plotz W, Rechl H, Burgkart R, Messmer C, Schelter R, Hipp E, et al. Limb salvage with tumor endoprostheses for malignant tumors of the knee. Clin Orthop Relat Res. 2002;207-215.

12. Abudu A, Grimer RJ, Tillman RM, Carter SR. Endoprosthetic replacement of the distal tibia and ankle joint for aggressive bone tumours. Int Orthop. 1999;23:291-4

13. Bishop AT, Wood MB, Sheetz KK. Arthrodesis of the ankle with a free vascularized autogenous bone graft. Reconstruction of segmental loss of bone secondary to osteomyelitis, tumor, or trauma. J Bone Joint Surg Am. 1995;77:1867-75

14. Niimi R, Matsumine A, Kusuzaki K, Kuratsu S, Araki N, Aoki Y, et al. Usefulness of limb salvage surgery for bone and soft tissue sarcomas of the distal lower leg. J Cancer Res Clin Oncol. 2008;134:1087-95.

15. Kawai A, Muschler GF, Lane JM, Otis JC, Healey JH. Prosthetic knee replacement after resection of a malignant tumor of the distal part of the femur. Medium to long-term results. J Bone Joint Surg Am. 1998;80:636-47.
16. Aho AJ, Ekfors T, Dean PB, Aro HT, Ahonen A, Nikkanen V. Incorporation and clinical results of large allografts of the extremities and pelvis. Clin Orthop Relat Res. 1994;307:200-13.

17. Hornicek Jr FJ, Mnaymneh W, Lackman RD, Exner GU, Malinin TI. Limb salvage with osteoarticular allografts after resection of proximal tibia bone tumors. Clin Orthop Relat Res. 1998;352:179-86.

18. Thompson Jr RC, Garg A, Clohisy DR, Cheng EY. Fractures in large-segment allografts. Clin Orthop Relat Res. 2000;370:227-35.

19. Ortiz-Cruz E, Gebhardt MC, Jennings LC, Springfield DS, Mankin HJ. The results of transplantation of intercalary allografts after resection of tumors. A long-term follow-up study. J Bone Joint Surg Am. 1997;79:97-106.

20. Rodl RW, Ozaki T, Hoffmann C, Bottner F, Lindner N, Winkelmann W. Osteoarticular allograft in surgery for high-grade malignant tumours of bone. J Bone Joint Surg Br. 2000;82:1006-10

21. Hornicek FJ, Gebhardt MC, Tomford WW, Sorger Jl, Zavatta M, Menzner JP, et al. Factors affecting nonunion of the allograft-host junction. Clin Orthop Relat Res. 2001;382:87-98.

22. Aronson J, Johnson E, Harp JH. Local bone transportation for treatment of intercalary defects by the llizarov technique. Biomechanical and clinical considerations. Clin Orthop Relat Res. 1989;243:71-9.

23. Li Z, Zhang X, Duan $L$, Chen $X$. Distraction osteogenesis technique using an intramedullary nail and a monolateral external fixator in the reconstruction of massive postosteomyelitis skeletal defects of the femur. Can J Surg. 2009;52:103-11.

24. Hayashi K, Tsuchiya H, Yamamoto N, Takeuchi A, Tomita K. Functional outcome in patients with osteosarcoma around the knee joint treated by minimised surgery. Int Orthop. 2008;32:63-8.

25. Tsuchiya H, Morsy AF, Matsubara H, Watanabe K, Abdel-Wanis ME, Tomita K. Treatment of benign bone tumours using external fixation. J Bone Joint Surg Br. 2007;89:1077-83.

26. Demiralp B, Ege T, Kose O, Yurttas Y, Basbozkurt M. Reconstruction of intercalary bone defects following bone tumor resection with segmental bone transport using an Ilizarov circular external fixator. J Orthop Sci. 2014;19:1004-11.

27. Kapukaya A, Subasi M, Kandiya E, Ozates M, Yilmaz F. Limb reconstruction with the callus distraction method after bone tumor resection. Arch Orthop Trauma Surg. 2000;120:215-8

28. McCoy Jr TH, Kim HJ, Cross MB, Fragomen AT, Healey JH, Athanasian EA, et al. Bone tumor reconstruction with the llizarov method. J Surg Oncol. 2013;107:343-52.

29. Tsuchiya H, Abdel-Wanis M, Kitano S, Sakurakichi K, Yamashiro T, Tomita K. The natural limb is best: joint preservation and reconstruction by distraction osteogenesis for high-grade juxta-articular osteosarcomas. Anticancer Res. 2002;22:2373-6

30. Shalaby S, Shalaby H, Bassiony A. Limb salvage for osteosarcoma of the distal tibia with resection arthrodesis, autogenous fibular graft and llizarov external fixator. J Bone Joint Surg Br. 2006;88:1642-6.

31. Casadei R, Ruggieri P, Giuseppe T, Biagini R, Mercuri M. Ankle resection arthrodesis in patients with bone tumors. Foot Ankle Int. 1994;15:242-9.

32. Campanacci DA, Scoccianti G, Beltrami G, Mugnaini M, Capanna R. Ankle arthrodesis with bone graft after distal tibia resection for bone tumors. Foot Ankle Int. 2008;29:1031-7.

33. Khattak MJ, Umer M, Haroon Ur R, Umar M. Autoclaved tumor bone for reconstruction: an alternative in developing countries. Clin Orthop Relat Res. 2006:447:138-44.

34. Cavadas PC, Landin L, Ibanez J, Roger I, Nthumba P. Infrapopliteal lower extremity replantation. Plast Reconstr Surg. 2009;124:532-9.

35. Hierner R, Berger AK, Frederix PR. Lower leg replantation-decision-making, treatment, and long-term results. Microsurgery. 2007;27:398-410.

36. Tsuchiya H, Tomita K. Distraction osteogenesis for treatment of bone loss in the lower extremity. J Orthop Sci. 2003;8:116-24.

37. El-Alfy B. Arthrodesis of the ankle joint by llizarov external fixator in patients with infection or poor bone stock. Foot Ankle Surg. 2010;16:96-100.

38. Tsuchiya H, Shirai T, Morsy AF, Sakayama K, Wada T, Kusuzaki K, et al. Safety of external fixation during postoperative chemotherapy. J Bone Joint Surg Br. 2008;90:924-8. 